\title{
SCALING LIMIT OF TWO-COMPONENT INTERACTING BROWNIAN MOTIONS
}

\author{
BY INSUK SEO \\ Seoul National University
}

\begin{abstract}
This paper presents our study of the asymptotic behavior of a twocomponent system of Brownian motions undergoing certain form of singular interactions. In particular, the system is a combination of two different types of particles and the mechanical properties and the interaction parameters depend on the corresponding type of particles. We prove that the hydrodynamic limit of the empirical densities of two types is the solution of a partial differential equation known as the Maxwell-Stefan equation.
\end{abstract}

1. Introduction. In this article, we are primarily concerned with the largescale analysis of the locally interacting Brownian motions (LIBM), which consists of two different types of particles. Originally, the LIBM consisting of identical particles was introduced by [10,11], in which the limit theorem for the tagged particle in nonequilibrium dynamics and the propagation of chaos was established. Recently, in [27] the author of the current paper developed a large deviation principle for the bulk average of particle trajectories of the one-component LIBM by analyzing the so-called two-color system. In the two-color system, all particles have the same mechanical property but each particle is painted by either black or white. The analysis of this system is known to be a difficult task because of the so-called nongradient property, and accordingly, the limit theorem and the large deviation principle for the two-color system are known only for three interacting particle systems: the symmetric simple exclusion process (SSEP) [24], the zerorange process (ZRP) [7] and the LIBM [27].

Furthermore, we can also consider the two-component system, instead of the two-color system, in which particles of different colors have different mechanical properties. Few results are announced for the two-component SSEP with additional interaction mechanisms. For instance, a two-component exclusion process where two types of particles affect each other through their jump rates is studied in [6]. A two-component SSEP under the presence of simultaneous births, deaths and switching of different types of particles is investigated in [26]. Recently, a twocomponent weakly asymmetric exclusion process where the type of each particle is randomly updated according to the types of its neighboring particles is analyzed

Received January 2016; revised February 2017.

MSC2010 subject classifications. 82C22, 60F10, 35K55, 35Q72.

Key words and phrases. Interacting Brownian motions, two-component system, hydrodynamic limit, strongly coupled parabolic systems, Maxwell-Stefan equation. 
in [5]. However, the hydrodynamic limit of the two-component SSEP without additional interaction mechanism other than the exclusion dynamics is an open problem. This kind of result is obtained for the ZRP. In [8], the hydrodynamic limit for the two-component ZRP without additional interaction mechanism other than zero-range dynamics is obtained. In this paper, we develop the second result of this kind for the two-component LIBM. We also provide extensive discussions for general features and technical difficulties for two-component interacting particle systems.

We also remark here that another two-component system under the recent attention is the chain of harmonic oscillators [21, 29]. For these models, two conserved quantities are the energy and the volume of the system.

1.1. Outline. In Section 2, we introduce a precise definition of the particle system and state our main results. We also discuss the main feature of the model, which enables us to obtain the hydrodynamic limit although the system is nongradient. In Section 3, we establish the hydrodynamic limit of the system, which amounts to the main result. The hydrodynamic limit equation is a system of parabolic equations (2.14) with the the explicit cross-diffusion matrix $D$ [cf. (2.15)], where the diffusion matrix $D$ is not symmetric, and $\frac{1}{2}\left(D+D^{\dagger}\right)$ is not even positive-definite. For this equation, the uniqueness of the weak solution is known to be a delicate problem, and the general theory [1] only provides the local uniqueness. Consequently, the hydrodynamic limit result for the general initial condition is local in time. The global result is achieved only for the initial condition that is sufficiently close to the equilibrium. This finding is a common feature of multi-component interacting particle systems, including the ZRP, due to the result of [8]. We discuss these uniqueness issues in Section 4. We finally remark here that the hydrodynamic limit equation (2.14) for the two-component LIBM is the so-called Maxwell-Stefan equation, which describes multi-component gaseous mixtures and is explained in Section 4.3.

\section{Model and main result.}

2.1. Type of particles. Consider a system of $N$ particles $x_{1}^{N}(\cdot), \ldots, x_{N}^{N}(\cdot)$ on $\mathbb{T}$ where each particle belongs to one of the two given types, namely, type 1 and type 2 . We denote by $\mathcal{T}_{c}^{N} \subset\{1,2, \ldots, N\}$, the index sets of type $c \in\{1,2\}$. Specifically, the set of particles of type $c \in\{1,2\}$ is $\left\{x_{i}^{N}(t): i \in \mathcal{T}_{c}^{N}\right\}$. The diffusivity of the particle of type $c$ is $\sigma_{c}^{2}>0$ and our primary interest is the nonhomogeneous case: $\sigma_{1}^{2} \neq \sigma_{2}^{2}$. If $\sigma_{1}^{2}=\sigma_{2}^{2}$, the system becomes the one-component, two-color system considered in [27].

In addition, the interaction mechanism also depends on the type of particles. In the local interaction model, two particles always reflect each other regardless of their types when they collide, but they sometimes change their labels. To explain 
this switching mechanism more precisely, we first measure the amount of collision between two particles $x_{i}^{N}(\cdot)$ and $x_{j}^{N}(\cdot)$ up to time $t$ by local times $A_{i j}^{N}(t)$ and $A_{j i}^{N}(t)$, depending on their relative positions infinitesimally before the collision:

$$
\begin{aligned}
& A_{i j}^{N}(t)=\lim _{\varepsilon \rightarrow 0} \int_{0}^{t} \frac{\mathbb{1}_{[0, \varepsilon]}\left(x_{i}^{N}(s)-x_{j}^{N}(s)\right)}{2 \varepsilon} d s, \\
& A_{j i}^{N}(t)=\lim _{\varepsilon \rightarrow 0} \int_{0}^{t} \frac{\mathbb{1}_{[-\varepsilon, 0]}\left(x_{i}^{N}(s)-x_{j}^{N}(s)\right)}{2 \varepsilon} d s,
\end{aligned}
$$

where the limit exists almost surely, for example; see [14], Chapter 2. In the onecomponent model $[10,27]$, the label switching between two particles $x_{i}^{N}(\cdot)$ and $x_{j}^{N}(\cdot)$ occurs as a Poisson process with constant intensity $\lambda N$ along this local time clock. The main difference for the two-component system is the dependence of the interaction parameter $\lambda$ on the types of particles involved. Let $\lambda_{c_{1}, c_{2}} \geq 0, c_{1}, c_{2} \in$ $\{1,2\}$, be four (possibly different) constants. Then the label switching between $x_{i}^{N}(\cdot)$ of type $c_{1}$ and $x_{j}^{N}(\cdot)$ of type $c_{2}$, occurs as the Poisson process with intensity $\lambda_{c_{1}, c_{2}} N$ along the local time $A_{i j}^{N}(t)$ and with intensity $\lambda_{c_{2}, c_{1}} N$ along the local time $A_{j i}^{N}(t)$. The rigorous definition of the model described above will be given in the next subsection.

REMARK 2.1. Our primary interest in the current paper is the symmetric case, that is, $\lambda_{1,2}=\lambda_{2,1}$, which shall be explicitly remarked in Section 2.3. On the other hand, our construction of the process presented in Section 2.2 is valid without this constraint.

2.2. Rigorous formulation of two-component LIBM. The rigorous construction of the model described above can be carried out in a similar manner to the one-component system [10,27]. Let $G_{N}$ be the $N$-manifold

$$
G_{N}=\left\{\mathbf{x}=\left(x_{1}, x_{2}, \ldots, x_{N}\right) \in \mathbb{T}^{N}: x_{i} \neq x_{j} \text { for all } i \neq j\right\},
$$

then the LIBM is constructed as a diffusion process on $G_{N}$ with reflecting boundary condition. We first characterize the boundary $\partial G_{N}$ of $G_{N}$. Any point $\mathbf{x} \in \partial G_{N}$ must satisfy $x_{i}=x_{j}$ for some $i \neq j$. However, the face $\left\{\mathbf{x}: x_{i}=x_{j}\right\}, i \neq j$, has two sides $F_{i j}$ and $F_{j i}$ corresponding to the boundary of two disconnected sets $U_{i j}=\left\{\mathbf{x} \in G_{N}: x_{i}<x_{j}\right\}$ and $U_{j i}=\left\{\mathbf{x} \in G_{N}: x_{j}<x_{i}\right\}$, respectively. Specifically, $F_{i j}, i \neq j$, can be regarded as the equivalence class on sequences $\left(\mathbf{x}_{n}\right)_{n=1}^{\infty}$ in $U_{i j}$ which converges to some point $\mathbf{x}$ satisfying $x_{i}=x_{j}$. The equivalent class $\sim$ is defined by $\left(\mathbf{x}_{n}\right)_{n=1}^{\infty} \sim\left(\widetilde{\mathbf{x}}_{n}\right)_{n=1}^{\infty}$ if $\lim \mathbf{x}_{n}=\lim \widetilde{\mathbf{x}}_{n}$. Then the boundary $\partial G_{N}$ can be written as $\partial G_{N}=\bigcup_{i \neq j} F_{i j}$. Denote by $\overline{\mathcal{C}}\left(G_{N}\right)$ the set of smooth functions on $G_{N}$ that are smooth up to the boundary $\partial G_{N}$. For $1 \leq i \leq N$, denote by $c(i)$ the type 
of particle $x_{i}^{N}(\cdot)$. For $f \in \overline{\mathcal{C}}\left(G_{N}\right)$ and $\mathbf{x} \in F_{i j}$, define

$$
\begin{aligned}
f_{i j}(\mathbf{x}) & =\lim _{\mathbf{x}_{n} \rightarrow \mathbf{x}, \mathbf{x}_{n} \in U_{i j}, \forall n} f\left(\mathbf{x}_{n}\right), \\
\left(D_{i j} f\right)(\mathbf{x}) & =\lim _{\mathbf{x}_{n} \rightarrow \mathbf{x}, \mathbf{x}_{n} \in U_{i j}, \forall n}\left(\sigma_{c(i)}^{2} \partial_{i}-\sigma_{c(j)}^{2} \partial_{j}\right) f\left(\mathbf{x}_{n}\right),
\end{aligned}
$$

so that $f_{i j}$ and $D_{i j} f$ are functions on $F_{i j}$.

The LIBM is a diffusion process $\mathbf{x}^{N}(\cdot)=\left(x_{1}^{N}(\cdot), \ldots, x_{N}^{N}(\cdot)\right)$ on $G_{N}$ with the pregenerator:

$$
\mathscr{L}_{N} f=\frac{\sigma_{1}^{2}}{2} \sum_{i \in \mathcal{T}_{1}^{N}} \partial_{i}^{2} f+\frac{\sigma_{2}^{2}}{2} \sum_{j \in \mathcal{T}_{2}^{N}} \partial_{j}^{2} f=\frac{1}{2} \sum_{i=1}^{N} \sigma_{c(i)}^{2} \partial_{i}^{2} f,
$$

where the domain $\mathcal{D}\left(\mathscr{L}_{N}\right)$ of $\mathscr{L}_{N}$ consists of functions $f \in \overline{\mathcal{C}}\left(G_{N}\right)$ satisfying the boundary condition $\left(\mathscr{B}_{i j} f\right)(\mathbf{x})=0$ on $F_{i j}$ for all $i \neq j$, where $\mathscr{B}_{i j} f$ is a function on $F_{i j}$ defined by

$$
\mathscr{B}_{i j} f=D_{i j} f-\lambda_{c(i), c(j)} N\left(f_{i j}-f_{j i}\right) .
$$

In (2.7), the first term corresponds to the reflection between two particles $x_{i}^{N}(\cdot)$ and $x_{j}^{N}(\cdot)$, while the second term explains the switching of labels between the two particles. The Lebesgue measure $d \mathbf{x}$ on $G_{N}$ is the invariant measure for $\mathscr{L}_{N}$, and the process $\mathbf{x}^{N}(\cdot)$ is reversible with respect to $d \mathbf{x}$.

Alternative construction of the particle system can be achieved by the martingale formulation of the diffusion processes reflecting at the boundary [13], as noted in [10]. More precisely, for the fixed final time $T>0$, the diffusion process $\mathbf{x}^{N}(\cdot)$ on $G_{N}$ that we constructed above can be regarded as a probability measure $\mathbb{P}_{N}$ on $C\left([0, T], G_{N}\right)$. Then there exist local times $A_{i j}^{N}(t), 1 \leq i \neq j \leq N$, such that, for all $f \in \overline{\mathcal{C}}\left(G_{N}\right)$,

$$
\begin{gathered}
f\left(\mathbf{x}^{N}(t)\right)-f\left(\mathbf{x}^{N}(0)\right)-\int_{0}^{t} \sum_{i=1}^{N} \frac{\sigma_{c(i)}^{2}}{2}\left(\partial_{i}^{2} f\right)\left(\mathbf{x}^{N}(s)\right) d s \\
-\sum_{i \neq j} \int_{0}^{t}\left(\mathscr{B}_{i j} f\right)\left(\mathbf{x}^{N}(s)\right) d A_{i j}^{N}(s)
\end{gathered}
$$

is a $\left(\mathbb{P}_{N},\left\{\mathscr{F}_{t}: 0 \leq t \leq T\right\}\right)$ martingale where $\mathscr{F}_{t}=\sigma\left(\mathbf{x}^{N}(s): 0 \leq s \leq t\right)$. The martingale (2.8) admits the following alternative expression:

$$
\begin{aligned}
\sum_{i=1}^{N} \sigma_{c(i)} & \int_{0}^{t}\left(\partial_{i} f\right)\left(\mathbf{x}^{N}(s)\right) d \beta_{i}(s) \\
& +\sum_{i \neq j} \int_{0}^{t}\left(f_{i j}-f_{j i}\right)\left(\mathbf{x}^{N}(s)\right)\left[d M_{i j}^{N}(s)-d M_{j i}^{N}(s)\right],
\end{aligned}
$$


where $\left\{\beta_{i}(\cdot): 1 \leq i \leq N\right\}$ is a family of independent Brownian motions and $\left\{M_{i j}^{N}(\cdot): 1 \leq i \neq j \leq N\right\}$ is a family of pairwise orthogonal compensated Poisson jump processes where the rate of process $M_{i j}^{N}(t)$ is $\lambda_{c(i), c(j)} N A_{i j}^{N}(t)$ for all $i \neq j$. We denote the expectation with respect to $\mathbb{P}_{N}$ as $\mathbb{E}_{N}$.

REMARK 2.2. The particle system consisting of $m$ different types $1,2, \ldots, m$ can be defined similarly.

2.3. Main result. The empirical density at time $t$ of particles of type $c \in\{1,2\}$ is defined by

$$
\mu_{c}^{N}(t)=\frac{1}{N} \sum_{i \in \mathcal{T}_{c}^{N}} \delta_{x_{i}^{N}(t)} \in \mathscr{M}(\mathbb{T}),
$$

and then the empirical density at time $t$ of the entire system can be written as

$$
\tilde{\mu}^{N}(t)=\left(\mu_{1}^{N}(t), \mu_{2}^{N}(t)\right)^{\dagger} \in \mathscr{M}(\mathbb{T})^{2} .
$$

Fix the final time $T$ and let $\mathbb{Q}_{N}$ be the measure on $C\left([0, T], \mathscr{M}(\mathbb{T})^{2}\right)$ induced by the process $\tilde{\mu}^{N}(\cdot)$. Then our goal is to characterize the limit point of $\left\{\mathbb{Q}_{N}\right\}_{N=1}^{\infty}$ as a Dirac measure on the unique solution of a certain partial differential equation (PDE). To specify the initial condition of the PDE, we assume that the initial empirical density $\widetilde{\mu}^{N}(0)$ satisfies the law of large number in the sense that

$$
\tilde{\mu}^{N}(0) \rightarrow \widetilde{\rho}^{0}(x) d x=\left(\rho_{1}^{0}(x) d x, \rho_{2}^{0}(x) d x\right)^{\dagger}
$$

weakly in $\mathscr{M}(\mathbb{T})^{2}$ for some nonnegative initial density functions $\rho_{1}^{0}$ and $\rho_{2}^{0}$. By pairing this weak convergence with the constant function 1 , we derive $\left|\mathcal{T}_{c}^{N}\right|=$ $N\left(\bar{\rho}_{c}+o_{N}(1)\right)$ where $\bar{\rho}_{c}=\int_{\mathbb{T}} \rho_{c}^{0}(x) d x$ is the average density of type $c$.

We explain several technical assumptions to obtain the result in a concrete form. The standard Sobolev space on $\mathbb{T}$ is denoted by $W_{k, p}(\mathbb{T})$, and let $H_{k}(\mathbb{T})=$ $W_{k, 2}(\mathbb{T})$. The following assumptions are required in the investigation of the uniqueness result in Section 4. The results in Section 3 are valid without these assumptions.

Assumption 2.1. The function $\widetilde{\rho}^{0}(\cdot)$ belongs to $W^{1, p}(\mathbb{T})^{2}$ for some $p>2$.

Assumption 2.2. The function $\widetilde{\rho}^{0}(\cdot)$ belongs to $H_{2}(\mathbb{T})^{2}$, and satisfies

$$
\left\|\rho_{1}^{0}(\cdot)-\bar{\rho}_{1}\right\|_{H_{2}(\mathbb{T})}+\left\|\rho_{2}^{0}(\cdot)-\bar{\rho}_{2}\right\|_{H_{2}(\mathbb{T})}<\varepsilon
$$

for some sufficiently small constant $\varepsilon=\varepsilon\left(\lambda, \sigma_{1}, \sigma_{2}\right)>0$. This constant is explained at the end of Section 4.3.

In addition, we also assume that the initial particle configuration satisfies the following entropy condition $[12,32]$. 
Assumption 2.3. The distribution of initial configuration $\mathbf{x}^{N}(0)$ is absolutely continuous with respect to the Lebesgue measure $d \mathbf{x}$, and the probability density function $f_{N}^{0}(\cdot)$ of $\mathbf{x}^{N}(0)$ satisfies

$$
\int_{G_{N}} f_{N}^{0}(\mathbf{x}) \log f_{N}^{0}(\mathbf{x}) d \mathbf{x} \leq C N \quad \forall N \in \mathbb{N},
$$

for some constant $C>0$ independent of $N$.

This assumption enables us to control the entropy production in Section 3.2 and is required in the proof of the tightness of $\left\{\mathbb{Q}_{N}\right\}_{N=1}^{\infty}$ in Section 3. Remark that the i.i.d. configuration satisfies Assumption 2.3.

In this paper, we are interested in the symmetric case, that is, $\lambda_{1,2}=\lambda_{2,1}$ only. Thus, for the sake of convenience, we shall normalize

$$
\lambda_{1,2}=\lambda_{2,1}=\lambda \sigma_{1}^{2} \sigma_{2}^{2} .
$$

In particular, we are concerned with the asymptotic behavior of the empirical density $\tilde{\mu}^{N}(\cdot)$, which is not affected by the switching of the labels between particles of the same type. Hence, the interactions among the particles of same type certainly do not affect $\widetilde{\mu}^{N}(\cdot)$ and in turn, neither the assumptions nor the conclusion are influenced even if we assume that the self-interaction parameters $\lambda_{1,1}$ and $\lambda_{2,2}$ satisfy

$$
\lambda_{1,1}=\lambda \sigma_{1}^{4}, \quad \lambda_{2,2}=\lambda \sigma_{2}^{4} .
$$

We emphasize that all the results obtained in this article are remaining in force for systems with any interacting parameters, $\lambda_{1,1}, \lambda_{2,2}>0$. Finally, we can write the interaction parameter between $x_{i}^{N}(\cdot)$ and $x_{j}^{N}(\cdot)$ by $\lambda \sigma_{c(i)}^{2} \sigma_{c(j)}^{2}$, under the notation (2.12) and (2.13).

The following theorem is the main result.

THEOREM 2.1. Under Assumption 2.3, the family of probability measures $\left\{\mathbb{Q}_{N}\right\}_{N=1}^{\infty}$ is tight and any of its limit point is concentrated on the trajectory of the form $\left(\rho_{1}(\cdot, x) d x, \rho_{2}(\cdot, x) d x\right)^{\dagger}$, where $\left(\rho_{1}, \rho_{2}\right)^{\dagger}$ is a weak solution of the equation

$$
\frac{\partial}{\partial t}\left(\begin{array}{l}
\rho_{1} \\
\rho_{2}
\end{array}\right)=\frac{1}{2} \nabla \cdot\left[D\left(\rho_{1}, \rho_{2}\right) \nabla\left(\begin{array}{l}
\rho_{1} \\
\rho_{2}
\end{array}\right)\right] ; \quad t \in[0, T]
$$

with initial condition $\widetilde{\rho}^{0}(\cdot)=\left(\rho_{1}^{0}(\cdot), \rho_{2}^{0}(\cdot)\right)^{\dagger}$, and the cross-diffusion matrix $D(\cdot, \cdot)$ is given by

$$
D\left(\rho_{1}, \rho_{2}\right)=\frac{1}{\lambda+\frac{\rho_{1}}{\sigma_{1}^{2}}+\frac{\rho_{2}}{\sigma_{2}^{2}}}\left(\begin{array}{cc}
\rho_{1}+\lambda \sigma_{1}^{2} & \rho_{1} \\
\rho_{2} & \rho_{2}+\lambda \sigma_{2}^{2}
\end{array}\right) .
$$




\section{Furthermore:}

1. (Local result). Under Assumption 2.1, there exists $t^{+}\left(\widetilde{\rho}^{0}\right)>0$ such that equation (2.14) has a unique weak solution in $\left[0, t^{+}\left(\widetilde{\rho}^{0}\right)\right)$. Therefore, $\left\{\mathbb{Q}_{N}\right\}_{N=1}^{\infty}$ converges weakly to the Dirac mass concentrated on this unique solution if $T \leq t^{+}\left(\widetilde{\rho}^{0}\right)$.

2. (Global result). Under Assumption 2.2, the weak solution of equation (2.14) is unique for any $T>0$. Hence, $\left\{\mathbb{Q}_{N}\right\}_{N=1}^{\infty}$ converges weakly to the Dirac mass concentrated on this unique solution.

We now briefly explain the main feature of the model which enables us to compute the hydrodynamic limit with the explicit diffusion coefficient (2.15). We first review the model with $\sigma_{1}=\sigma_{2}=1$, which is the one-component, two-color system considered in [27]. In general, the two-color interacting particle system is nongradient in the sense of [32], and this property makes the analysis of hydrodynamic limit a complicated project. The robust non-gradient method has been developed by Varadhan [32], and its application to the two-color SSEP was achieved by Quastel [24]. For the detailed discussion on the nongradient method, we refer to [20], Chapter 7.

The one-component LIBM is verified to be a nongradient system. Define $\langle\mu, f\rangle=\int_{\mathbb{T}} f d \mu$. Then the so-called density field for type $c \in\{1,2\}$ corresponding to the function $g \in C^{1}(\mathbb{T})$ is defined by $\left\langle\mu_{c}^{N}(t), g\right\rangle$. Then, by (2.8), we can observe that $\left\langle\mu_{c}^{N}(t), g\right\rangle-\left\langle\mu_{c}^{N}(0), g\right\rangle$ is equal to

$$
\frac{1}{N} \sum_{i \in \mathcal{T}_{1}^{N}} \int_{0}^{t} g^{\prime}\left(x_{i}^{N}(s)\right) \cdot N \sum_{k: k \neq i}\left[d A_{i k}^{N}(s)-d A_{k i}^{N}(s)\right]+M_{t},
$$

where $M_{t}$ is a martingale. Hence, in view of (2.1) and (2.2), the current around the particle $x_{i}^{N}(s)$ can be formally defined by

$$
g^{\prime}\left(x_{i}^{N}(s)\right) \cdot N \sum_{k: k \neq i}\left[\delta_{+}\left(x_{k}^{N}(s)-x_{i}^{N}(s)\right)-\delta_{-}\left(x_{i}^{N}(s)-x_{k}^{N}(s)\right)\right],
$$

where $\delta_{+}$and $\delta_{-}$are right and left Dirac functions at 0 , respectively. The LIMB can be easily observed as a nongradient system in this expression. The application of the nongradient method to the singular object, such as (2.16), became a technically difficult issue. Furthermore, at the time when this paper was written, the nongradient method could not be applied to the nongradient, diffusion-type interacting particle system. For instance, the analysis of the two-color system of interacting diffusions considered in [31] is an open problem. However, the LIBM owns a particular feature, which enables us to derive the hydrodynamic limit without appealing the non-gradient method. This feature is briefly explained below.

In [19], Kipnis and Varadhan reduced the investigation of the tagged particle in a reversible interacting particle system to a central limit theorem for certain reversible Markov chains. We refer to [20], Chapter 6, for the detailed exposition 
of this topic. This central limit theorem is obvious if there exists a solution of a certain Poisson equation of the form $-\mathcal{L} f=V$, for example, see [20], Chapter 1 , for details. The general methodology of [19] deals with the situation for which this Poisson equation does not have a solution. The main feature of the one-component LIBM is the explicit, simple solution in the corresponding Poisson equation. Hence, the argument of [19] is not required. Given this feature, we can simplify the computation considerably and compute the diffusion coefficient in an explicit form. Furthermore, this feature is also useful in the examination of the nonequilibrium tagged particle in [10]. More precisely, in [10], Definition 8 , a martingale $z_{1}^{N}(t)$ is introduced and is constructed by using the explicit solution of the Poisson equation. The martingale $z_{1}^{N}(t)$ allows [10] and [27] to detour the nongradient method. Although our model is two-component, we introduce another form of martingale (3.12) in the computation of hydrodynamic limits. This martingale enables us to compute the explicit diffusion coefficient (2.15).

3. Hydrodynamic limit. In this section, we prove Theorem 2.1. The uniqueness issue pertaining to equation (2.14) is discussed in the next section.

3.1. Green's formula for $G_{N}$. We briefly review Green's formula for $G_{N}$. Although the current paper is self-contained, we refer to [27], Section 2.2, for detailed explanation.

Fix a function $u \in \overline{\mathcal{C}}\left(G_{N}\right)$ and a smooth vector field $\mathbf{V}$ on $G_{N}$. Recall that the boundary $\partial G_{N}$ can be expressed as $\bigcup_{i \neq j} F_{i j}$. Note that the normal vector to $F_{i j}$ is $\left(\mathbf{e}_{i}-\mathbf{e}_{j}\right) / \sqrt{2}$ where $\mathbf{e}_{i}$ represents the $i$ th standard normal vector. The Lebesgue measure on the surface $F_{i j}=\left\{\mathbf{x}: x_{i}=x_{j}\right\}$, which is denoted by $d S_{i j}(\mathbf{x})$, is normalized to have a total measure of 1 . Then Green's formula for $G_{N}$ implies that

$$
\begin{aligned}
\int_{G_{N}} \nabla u(\mathbf{x}) \cdot \mathbf{V}(\mathbf{x}) d \mathbf{x}= & -\int_{G_{N}} u(\mathbf{x})(\nabla \cdot \mathbf{V})(\mathbf{x}) d \mathbf{x} \\
& +\sum_{i \neq j} \int_{F_{i j}} u_{i j}(\mathbf{x})\left[\mathbf{V}(\mathbf{x}) \cdot\left(\mathbf{e}_{i}-\mathbf{e}_{j}\right)\right] d S_{i j}(\mathbf{x}) .
\end{aligned}
$$

Note that factor $1 / \sqrt{2}$ disappeared because of the renormalization of the measure $d S_{i j}$ on $F_{i j}$. This formula will be used frequently hereafter.

3.2. Entropy production. Since the invariant measure for $\mathscr{L}_{N}$ is $d \mathbf{x}$, the corresponding Dirichlet form is defined by $\mathscr{D}_{N}(f)=\int_{G_{N}} f(\mathbf{x})\left(-\mathscr{L}_{N} f\right)(\mathbf{x}) d \mathbf{x}$ for $f \in \overline{\mathcal{C}}\left(G_{N}\right)$. For nonnegative function $f \in \overline{\mathcal{C}}\left(G_{N}\right)$ define $\mathcal{D}_{N}(f)=\mathscr{D}_{N}(\sqrt{f})$. 
Then it is easy to verify that

$$
\begin{aligned}
\mathcal{D}_{N}(f)= & \sum_{i=1}^{N} \int_{G_{N}} \frac{\sigma_{c(i)}^{2}}{8} \frac{\left(\partial_{i} f\right)^{2}}{f} d \mathbf{x} \\
& +\sum_{i \neq j} \frac{\lambda N \sigma_{c(i)}^{2} \sigma_{c(j)}^{2}}{2} \int_{F_{i j}}\left(\sqrt{f_{i j}}-\sqrt{f_{j i}}\right)^{2} d S_{i j}(\mathbf{x}) .
\end{aligned}
$$

Denote by $f_{N}(t, \cdot)$ the marginal density of $\mathbf{x}^{N}(\cdot)$ at time $t$ with respect to $d \mathbf{x}$. Then the entropy at time $t$ is defined by

$$
H_{N}(t)=\int_{G_{N}} f_{N}(t, \mathbf{x}) \log f_{N}(t, \mathbf{x}) d \mathbf{x} .
$$

Proposition 3.1. We have that $\frac{d}{d t} H_{N}(t) \leq-4 \mathcal{D}_{N}\left(f_{N}(t, \cdot)\right)$.

PROOF. Since the function $f_{N}$ solves the equation $\partial_{t} f_{N}=(1 / 2) \Delta f_{N}$ on $G_{N}$, and satisfies the boundary condition $\mathscr{B}_{i j} f_{N} \equiv 0$ on $F_{i j}$ for all $i \neq j$, we obtain

$$
\begin{aligned}
\frac{d}{d t} H_{N}(t) & =\int_{G_{N}} \partial_{t} f_{N}(t, \mathbf{x}) \log f_{N}(t, \mathbf{x}) d \mathbf{x} \\
& =\int_{G_{N}} \Delta f_{N}(t, \mathbf{x}) \log f_{N}(t, \mathbf{x}) d \mathbf{x} .
\end{aligned}
$$

Hence, by (3.1) with $u(\mathbf{x})=\log f_{N}(t, \mathbf{x})$ and $\mathbf{V}(\mathbf{x})=\nabla f_{N}(t, \mathbf{x})$, we can rewrite $(d / d t) H_{N}(t)$ as

$$
\begin{aligned}
& -\sum_{i=1}^{N} \int_{G_{N}} \frac{\sigma_{c(i)}^{2}}{2} \frac{\left|\partial_{i} f_{N}(t, \mathbf{x})\right|^{2}}{f_{N}(t, \mathbf{x})} d \mathbf{x} \\
& \quad-\sum_{i \neq j} \frac{\lambda N \sigma_{c(i)}^{2} \sigma_{c(j)}^{2}}{2} \int_{F_{i j}}\left(\left(f_{N}\right)_{i j}-\left(f_{N}\right)_{j i}\right) \log \frac{\left(f_{N}\right)_{i j}}{\left(f_{N}\right)_{j i}}(t, \mathbf{x}) d S_{i j}(\mathbf{x}) .
\end{aligned}
$$

The proof is completed by the elementary inequality

$$
(a-b) \log (a / b) \geq 4(\sqrt{a}-\sqrt{b})^{2} .
$$

For $t_{1}<t_{2}$, define $\bar{f}_{N}^{\left[t_{1}, t_{2}\right]}(\mathbf{x})=\left(t_{2}-t_{1}\right)^{-1} \int_{t_{1}}^{t_{2}} f_{N}(t, \mathbf{x}) d t$, and denote $\bar{f}_{N}^{[0, T]}$ simply by $\bar{f}_{N}$.

COROLlary 3.1. For all $0 \leq t_{1}<t_{2} \leq T$, we have that

$$
\mathcal{D}_{N}\left(\bar{f}_{N}^{\left[t_{1}, t_{2}\right]}\right) \leq C N /\left(t_{2}-t_{1}\right),
$$

where the constant $C$ does not depend on $t_{1}, t_{2}$ and $N$. 
Proof. By the convexity of the Dirichlet form,

$$
\mathcal{D}_{N}\left(\bar{f}_{N}^{\left[t_{1}, t_{2}\right]}\right) \leq \frac{1}{t_{2}-t_{1}} \int_{t_{1}}^{t_{2}} \mathcal{D}_{N}\left(f_{N}(t, \cdot)\right) d t .
$$

By Proposition 3.1 and Assumption 2.3, the right-hand side of the above inequality is bounded above by

$$
-\frac{1}{4\left(t_{2}-t_{1}\right)} \int_{t_{1}}^{t_{2}} \frac{d}{d t} H_{N}(t) d t=\frac{H_{N}\left(t_{1}\right)-H_{N}\left(t_{2}\right)}{4\left(t_{2}-t_{1}\right)} \leq \frac{H_{N}(0)}{4\left(t_{2}-t_{1}\right)} \leq \frac{C N}{t_{2}-t_{1}} .
$$

3.3. Tightness. Denote by $\mathbb{P}_{N}^{\mathrm{eq}}$ the equilibrium process starting from the invariant measure $d \mathbf{x}$. We recall the following Dirichlet form for the one-component system [27] with the interaction parameter $\lambda>0$ :

$$
\widetilde{\mathcal{D}}_{N}(f)=\frac{1}{8} \sum_{i=1}^{N} \int_{G_{N}} \frac{\left(\partial_{i} f\right)^{2}}{f} d \mathbf{x}+\frac{\lambda N}{2} \sum_{i \neq j} \int_{F_{i j}}\left(\sqrt{f_{i j}}-\sqrt{f_{j i}}\right)^{2} d S_{i j}(\mathbf{x}) .
$$

Note that this Dirichlet form is equivalent to that in our study in the sense that

$$
C_{1} \mathcal{D}_{N}(f) \leq \widetilde{\mathcal{D}}_{N}(f) \leq C_{2} \mathcal{D}_{N}(f),
$$

where $C_{1}>0$ and $C_{2}>0$ are constants that depend only on $\sigma_{1}$ and $\sigma_{2}$. In [27], Proposition 3.4, the exponential tightness of the one-component LIBM in equilibrium has been developed, and the proof therein is entirely based on the estimates in terms of the Dirichlet form $\widetilde{\mathcal{D}}_{N}(\cdot)$. Accordingly, all the arguments are still valid for our model through equivalence (3.5). In this manner, we obtain the following tightness result for the equilibrium processes.

Proposition 3.2. For all $\varepsilon, \alpha>0$,

$$
\limsup _{\delta \rightarrow 0} \limsup _{N \rightarrow \infty} \frac{1}{N} \log \mathbb{P}_{N}^{\mathrm{eq}}\left[\left|\left\{i: \sup _{\substack{0 \leq s, t \leq T \\|s-t| \leq \delta}}\left|x_{i}^{N}(t)-x_{i}^{N}(s)\right| \geq \varepsilon\right\}\right| \geq N \alpha\right]=-\infty .
$$

This super-exponential estimate for the equilibrium processes allows us to develop the tightness of the nonequilibrium processes $\mathbb{P}_{N}$, as follows.

COROLlaRY 3.2. For all $\varepsilon, \alpha>0$,

$$
\limsup _{\delta \rightarrow 0} \limsup _{N \rightarrow \infty} \mathbb{P}_{N}\left[\left|\left\{i: \sup _{\substack{0 \leq s, t \leq T \\|s-t| \leq \delta}}\left|x_{i}^{N}(t)-x_{i}^{N}(s)\right| \geq \varepsilon\right\}\right| \geq N \alpha\right]=0 .
$$

In particular, $\left\{\mathbb{Q}_{N}\right\}_{N=1}^{\infty}$ is a tight family in $C\left([0, T], \mathscr{M}(\mathbb{T})^{2}\right)$. 
PROOF. Denote by $E_{N, \delta, \varepsilon, \alpha}$ the event inside the bracket of (3.6). By [18], Proposition 8.2 of Appendix 1, and by Assumption 2.3,

$$
\mathbb{P}_{N}\left[E_{N, \delta, \varepsilon, \alpha}\right] \leq \frac{2+H\left[\mathbb{P}_{N} \mid \mathbb{P}_{N}^{\mathrm{eq}}\right]}{\log \left(1+\mathbb{P}_{N}^{\mathrm{eq}}\left[E_{N, \delta, \varepsilon, \alpha}\right]^{-1}\right)} \leq \frac{2+C N}{-\log \mathbb{P}_{N}^{\mathrm{eq}}\left[E_{N, \delta, \varepsilon, \alpha}\right]}
$$

Hence, (3.6) is a consequence of Proposition 3.2.

We next prove the tightness of $\left\{\mathbb{Q}_{N}\right\}_{N=1}^{\infty}$. It suffices to demonstrate that, for $\varepsilon>0$ and $c \in\{1,2\}$,

$$
\lim _{\delta \rightarrow 0} \limsup _{N \rightarrow \infty} \mathbb{P}_{N}\left[\sup _{0 \leq s, t \leq T,|s-t| \leq \delta} \frac{1}{N} \sum_{i \in \mathcal{T}_{c}^{N}}\left|x_{i}^{N}(t)-x_{i}^{N}(s)\right| \geq \varepsilon\right]=0 .
$$

This estimate follows from (3.6), since the last probability is bounded above by

$$
\mathbb{P}_{N}\left[\left|\left\{i: \sup _{0 \leq s, t \leq T,|s-t| \leq \delta}\left|x_{i}^{N}(t)-x_{i}^{N}(s)\right| \geq \varepsilon / 2\right\}\right| \geq N \varepsilon / 2\right] .
$$

3.4. Energy estimate. We now establish an energy estimate. Let $\rho=\rho_{1}+\rho_{2}$.

Proposition 3.3. Suppose that $\mathbb{Q}_{\infty}$ is a weak limit of $\left\{\mathbb{Q}_{N}\right\}_{N=1}^{\infty}$. Then $\mathbb{Q}_{\infty}$ is concentrated on the trajectory of the form $\left(\rho_{1}(\cdot, x) d x, \rho_{2}(\cdot, x) d x\right)^{\dagger}$ for some $\rho_{1}, \rho_{2} \in L^{2}([0, T] \times \mathbb{T})$, which are weakly differentiable in $x$, and satisfy

$$
\int_{0}^{T} \int_{\mathbb{T}} \frac{\rho_{x}^{2}(t, x)}{\rho(t, x)} d x d t<\infty .
$$

PROOF. We claim first that all the conclusions of proposition follow from the following estimate for all $\phi \in C^{0,1}([0, T] \times \mathbb{T})$ :

$$
\mathbb{E}_{N}\left[\int_{0}^{T} \frac{1}{N} \sum_{i=1}^{N} \phi^{\prime}\left(x_{i}^{N}(t)\right) d t\right] \leq C \mathbb{E}_{N}\left[\int_{0}^{T} \frac{1}{N} \sum_{i=1}^{N} \phi^{2}\left(x_{i}^{N}(t)\right) d t\right]^{1 / 2} .
$$

Indeed, it is standard, for example, [18], Section 5.7, that (3.9) implies that $\rho$ is not only absolutely continuous with respect to the Lebesgue measure but also has a weak derivative $\rho_{x}$ that satisfies (3.8). By [27], Lemma 4.3, this energy estimate implies $\rho \in L^{2}([0, T] \times \mathbb{T})$ and accordingly $\rho_{1}, \rho_{2} \in L^{2}([0, T] \times \mathbb{T})$.

We now prove (3.9). By (3.1) with $u(\mathbf{x})=\phi\left(x_{1}\right)+\cdots+\phi\left(x_{N}\right)$ and $\mathbf{V}=$ $\left(\bar{f}_{N}, \ldots, \bar{f}_{N}\right)$, the left-hand side of (3.9) becomes

$$
\frac{T}{N} \int_{G_{N}} \sum_{i=1}^{N} \phi^{\prime}\left(x_{i}\right) \bar{f}_{N}(\mathbf{x}) d \mathbf{x}=-\frac{T}{N} \int_{G_{N}} \sum_{i=1}^{N} \phi\left(x_{i}\right)\left(\partial_{i} \bar{f}_{N}\right)(\mathbf{x}) d \mathbf{x} .
$$

By Cauchy-Schwarz's inequality, the right-hand side of (3.10) is bounded above by

$$
\frac{T}{N}\left[\int_{G_{N}} \sum_{i=1}^{N} \phi^{2}\left(x_{i}\right) \bar{f}_{N}(\mathbf{x}) d \mathbf{x} \int_{G_{N}} \sum_{i=1}^{N} \frac{\left(\partial_{i} \bar{f}_{N}\right)^{2}(\mathbf{x})}{\bar{f}_{N}(\mathbf{x})} d \mathbf{x}\right]^{1 / 2} .
$$

Thus, (3.9) follows from Corollary 3.1. 
3.5. Auxiliary martingales. Recall the average density $\bar{\rho}_{c}=\int_{\mathbb{T}} \rho_{c}^{0}(x) d x, c \in$ $\{1,2\}$, and define a constant by

$$
\alpha=\left(\lambda+\frac{\bar{\rho}_{1}}{\sigma_{1}^{2}}+\frac{\bar{\rho}_{2}}{\sigma_{2}^{2}}\right)^{-1} .
$$

Define $v(x)=x, x \in[0,1)$ and regard $v(\cdot)$ as a discontinuous function on $\mathbb{T}$. The process $z_{k}^{N}(t), 1 \leq k \leq N$, is defined by

$$
z_{k}^{N}(t)=x_{k}^{N}(t)+\frac{\alpha}{N} \sum_{i=1}^{N} \frac{1}{\sigma_{c(i)}^{2}} v\left(x_{i}^{N}(t)-x_{k}^{N}(t)\right) .
$$

We claim that $z_{k}^{N}(t)-z_{k}^{N}(0)$ is a martingale with respect to the filtration $\left\{\mathscr{F}_{t}: 0 \leq\right.$ $t \leq T\}$. To demonstrate this, define a function $r_{N, k}: G_{N} \rightarrow \mathbb{R}$ by

$$
r_{N, k}(\mathbf{x})=x_{k}+\frac{\alpha}{N} \sum_{i: i \neq k} \frac{1}{\sigma_{c(i)}^{2}} v\left(x_{i}-x_{k}\right) \text {. }
$$

Since $r_{N, k}$ is a linear function on $G_{N}$, it is obvious that $\partial_{i}^{2} r_{N, k} \equiv 0$ on $G_{N}$ for all $1 \leq i \leq N$, and it is also straightforward to check that $\mathscr{B}_{i j} r_{N, k} \equiv 0$ on $F_{i j}$ for all $1 \leq i \neq j \leq N$. Hence, by $(2.8), z_{k}^{N}(t)-z_{k}^{N}(0)=r_{N, k}\left(\mathbf{x}^{N}(t)\right)-r_{N, k}\left(\mathbf{x}^{N}(0)\right)$ is a martingale. Moreover, by (2.9), this martingale can be written as

$$
\begin{aligned}
z_{k}^{N}(t)-z_{k}^{N}(0)= & \alpha \lambda \sigma_{c(k)} \beta_{k}(t)+\frac{\alpha}{N} \sum_{i=1}^{N} \frac{1}{\sigma_{c(i)}} \beta_{i}(t) \\
& +\frac{\alpha}{N} \sum_{i: i \neq k} \frac{1}{\sigma_{c(i)}^{2}}\left[M_{i k}^{N}(t)-M_{k i}^{N}(t)\right]
\end{aligned}
$$

For $1 \leq i \leq N$ and $c \in\{1,2\}$, define the averaged local time $A_{i, c}^{N}(t)$ by

$$
A_{i, c}^{N}(t)=\frac{1}{N} \sum_{j \in \mathcal{T}_{c}^{N} \backslash\{i\}}\left[A_{i j}^{N}(t)+A_{j i}^{N}(t)\right] .
$$

Then the quadratic variation of the martingale $z_{k}^{N}(t)-z_{k}^{N}(0)$ can be written as

$$
\left\langle z_{k}^{N}, z_{k}^{N}\right\rangle_{t}=\lambda \alpha^{2} \sigma_{c(k)}^{2}\left[\lambda t+\frac{1}{\sigma_{1}^{2}} A_{k, 1}^{N}(t)+\frac{1}{\sigma_{2}^{2}} A_{k, 2}^{N}(t)\right]+O\left(N^{-1}\right) t .
$$

3.6. Mollification of local times. In the derivation of the hydrodynamic limit in the spirit of $[12,32]$, the evolution of the density field must be analyzed and the major technical issue in this investigation is the approximation of the interaction terms by a function of the empirical density. This step is known as the replacement lemma, and we refer to [18], Section 5, for the detailed exposition. In the context of this work, this interaction term is $J_{2}$ in (3.32) and, therefore, in view of (3.16), we 
must replace $A_{i, c}^{N}(t)$ with a function of the density field. To this end, we introduce the local density of particle configuration. Fix $\varepsilon>0$ and let $\iota_{\varepsilon}=(2 \varepsilon)^{-1} \mathbb{1}_{[-\varepsilon, \varepsilon]}$ be a function on $\mathbb{T}$. Then, for $\mathbf{x} \in G_{N}$, we define the local density of type $c \in\{1,2\}$ around $x_{i}, 1 \leq i \leq N$, by

$$
\rho_{i, c}^{N, \varepsilon}(\mathbf{x})=\frac{1}{N} \sum_{j \in \mathcal{T}_{c}^{N}} \iota_{\varepsilon}\left(x_{j}-x_{i}\right) .
$$

In view of (2.2), the local density $\rho_{i, c}^{N, \varepsilon}(\mathbf{x})$ is the natural candidate for the required replacement, and the corresponding approximation can be formally stated as follows.

THEOREM 3.1. For all $c_{1}, c_{2} \in\{1,2\}, \delta>0,0 \leq t_{1}<t_{2} \leq T$, and $h(\cdot, \cdot) \in$ $C^{1}([0, T] \times \mathbb{T})$, we have that

$$
\begin{aligned}
& \underset{\varepsilon \rightarrow 0}{\limsup } \limsup _{N \rightarrow \infty} \mathbb{P}_{N}\left[\left|\frac{1}{N} \sum_{i \in \mathcal{T}_{c_{1}}^{N}} \int_{0}^{T} h\left(t, z_{i}^{N}(t)\right)\left[d A_{i, c_{2}}^{N}(t)-\rho_{i, c_{2}}^{N, \varepsilon}\left(\mathbf{x}^{N}(t)\right) d t\right]\right|>\delta\right] \\
& \quad=0 .
\end{aligned}
$$

We now prove this theorem by several steps. Define

$$
\mathscr{P}_{N}=\left\{f \in \overline{\mathcal{C}}\left(G_{N}\right): \int_{G_{N}} f(x) d x=1\right\},
$$

and for $\varepsilon>0$ and $1 \leq i \leq N$, define

$$
M_{\varepsilon, i}(\mathbf{x})=\sum_{j: j \neq i} \mathbb{1}_{[-\varepsilon, \varepsilon]}\left(x_{j}-x_{i}\right)
$$

The following lemma was proven in [27], Lemmata 2.6, 2.7 and 2.10.

LemMA 3.1. For $f \in \mathscr{P}_{N}$ and $\varepsilon \in(0,1 / 4)$, we have that

$$
\begin{gathered}
\frac{1}{N^{2}} \sum_{i=1}^{N} \int_{G_{N}} f(\mathbf{x}) M_{\varepsilon, i}(\mathbf{x}) d \mathbf{x} \leq C \varepsilon\left[1+\left(\widetilde{\mathcal{D}}_{N}(f) / N\right)^{1 / 2}\right], \\
\frac{1}{N^{2}} \sum_{i=1}^{N} \int_{G_{N}}\left|\left(\partial_{i} f\right)(\mathbf{x})\right| M_{\varepsilon, i}(\mathbf{x}) d \mathbf{x} \leq C \varepsilon^{1 / 2}\left[1+\left(\widetilde{\mathcal{D}}_{N}(f) / N\right)^{3 / 4}\right],
\end{gathered}
$$

and finally,

$$
\begin{aligned}
& \frac{1}{N^{2}} \sum_{i \neq j} \int_{F_{i j}}\left|f_{i j}(\mathbf{x})-f_{j i}(\mathbf{x})\right| M_{\varepsilon, i}(\mathbf{x}) d S_{i j}(\mathbf{x}) \\
& \quad \leq C\left(\varepsilon^{1 / 4}+N^{-1 / 2}\right)\left[1+\left(\widetilde{\mathcal{D}}_{N}(f) / N\right)^{7 / 8}\right] .
\end{aligned}
$$

Furthermore, these estimates are still valid if we replace $\widetilde{\mathcal{D}}_{N}$ with $\mathcal{D}_{N}$. 
The proof of these estimates are entirely based on Green's formula (3.1), and we refer to [27], Section 2.2, for the detailed proofs of these estimates. Note that we can replace $\widetilde{\mathcal{D}}_{N}$ with $\mathcal{D}_{N}$ because of (3.5).

Let $z_{k}=r_{N, k}(\mathbf{x}), 1 \leq k \leq N$, where $r_{N, k}$ is defined in (3.13). The following lemma is a generalization of [27], Proposition 2.11.

Lemma 3.2. For all $f \in \mathscr{P}_{N}, h \in C^{1}(\mathbb{T})$ and $0<\varepsilon<\frac{1}{4}$, we have that

$$
\begin{aligned}
& \frac{1}{N^{2}} \sum_{i \neq j}\left|\int_{G_{N}} h\left(z_{i}\right) f(\mathbf{x}) \iota_{\varepsilon}\left(x_{j}-x_{i}\right) d \mathbf{x}-\frac{1}{2} \int_{F_{i j}} h\left(z_{i}\right)\left(f_{i j}+f_{j i}\right)(\mathbf{x}) d S_{i j}(\mathbf{x})\right| \\
& \quad \leq C\left(1+\left[\mathcal{D}_{N}(f) / N\right]^{\frac{7}{8}}\right)\left(\varepsilon^{\frac{1}{4}}+N^{-\frac{1}{2}}\right)
\end{aligned}
$$

for some constant $C=C(h)>0$ that only depends on $h$.

PROOF. Define a function $g_{\varepsilon}$ on $[0,1)$ by

$$
g_{\varepsilon}(x)=\left(\frac{x}{2 \varepsilon}-\frac{1}{2}\right) \mathbb{1}_{[0, \varepsilon]}(x)+\left(\frac{x-1}{2 \varepsilon}+\frac{1}{2}\right) \mathbb{1}_{[1-\varepsilon, 1)}(x)
$$

and regard $g_{\varepsilon}$ as a discontinuous function on $\mathbb{T}$. Fix $i \neq j$ and define a vector field $\mathbf{V}_{i j}(\mathbf{x})=h\left(z_{i}\right) g_{\varepsilon}\left(x_{i}-x_{j}\right) \mathbf{e}_{j}$ which is continuous on $G_{N}$. Fix $f \in \mathscr{P}_{N}$. By (3.1) with $u=f$, and $\mathbf{V}=\mathbf{V}_{i j}$, we obtain

$$
\int_{G_{N}}\left(\partial_{j} f\right)(\mathbf{x})\left[h\left(z_{i}\right) g_{\varepsilon}\left(x_{i}-x_{j}\right)\right] d \mathbf{x}=K_{i j}^{(1)}+K_{i j}^{(2)},
$$

where

$$
\begin{aligned}
K_{i j}^{(1)}= & \int_{G_{N}} f(\mathbf{x}) \partial_{j}\left[h\left(z_{i}\right) g_{\varepsilon}\left(x_{i}-x_{j}\right)\right] d \mathbf{x}, \\
K_{i j}^{(2)}= & \int_{F_{j i}} f_{j i}(\mathbf{x}) h\left(z_{i}\right) g_{\varepsilon}\left(x_{i}-x_{j}\right) d S_{j i}(\mathbf{x})-\int_{F_{i j}} f_{i j}(\mathbf{x}) h\left(z_{i}\right) g_{\varepsilon}\left(x_{i}-x_{j}\right) d S_{i j}(\mathbf{x}) \\
& +\sum_{k: k \neq i, j}\left[\int_{F_{j k}} f_{j k}(\mathbf{x}) h\left(z_{i}\right) g_{\varepsilon}\left(x_{i}-x_{j}\right) d S_{j k}(\mathbf{x})\right. \\
& \left.-\int_{F_{k j}} f_{k j}(\mathbf{x}) h\left(z_{i}\right) g_{\varepsilon}\left(x_{i}-x_{j}\right) d S_{k j}(\mathbf{x})\right] .
\end{aligned}
$$

Note that $K_{i j}^{(1)}$ and $K_{i j}^{(2)}$ correspond to the first and second terms of the right-hand side of (3.1), respectively. By simple computations, we deduce

$$
K_{i j}^{(1)}=\int_{G_{N}} f(\mathbf{x})\left[\frac{\alpha}{N \sigma_{c(j)}^{2}} h^{\prime}\left(z_{i}\right) g_{\varepsilon}\left(x_{i}-x_{j}\right)+h\left(z_{i}\right) \iota_{\varepsilon}\left(x_{i}-x_{j}\right)\right] d \mathbf{x} .
$$


Since $g_{\varepsilon}\left(x_{i}-x_{j}\right)=\frac{1}{2}$ on $F_{i j}$ and $-\frac{1}{2}$ on $F_{j i}$, and since $h\left(z_{i}\right) g_{\varepsilon}\left(x_{i}-x_{j}\right)$ has same value on $F_{j k}$ and $F_{k j}$ for $k \neq i, j$, we can simplify $K_{i j}^{(2)}$ to

$$
\begin{aligned}
K_{i j}^{(2)}= & -\frac{1}{2} \int_{F_{i j}} h\left(z_{i}\right)\left(f_{i j}(\mathbf{x})+f_{j i}(\mathbf{x})\right) d S_{i j}(\mathbf{x}) \\
& +\sum_{k: k \neq i, j}\left[\int_{F_{j k}}\left(f_{j k}(\mathbf{x})-f_{k j}(\mathbf{x})\right) h\left(z_{i}\right) g_{\varepsilon}\left(x_{i}-x_{j}\right) d S_{j k}(\mathbf{x})\right] .
\end{aligned}
$$

By (3.19), (3.20), (3.21) and by an elementary inequality $\left|g_{\varepsilon}\right| \leq \frac{1}{2} \chi_{\varepsilon}$, we can bound the left-hand side of (3.18) by

$$
\begin{aligned}
& \frac{C(h)}{N^{2}} \sum_{i=1}^{N} \int_{G_{N}}\left|\partial_{i} f(\mathbf{x})\right| M_{\varepsilon, i}(\mathbf{x}) d \mathbf{x}+\frac{C}{N^{3}} \sum_{i=1}^{N} \int_{G_{N}} f(\mathbf{x}) M_{\varepsilon, i}(\mathbf{x}) d \mathbf{x} \\
& \quad+\frac{C(h)}{N^{2}} \sum_{i \neq j} \int_{F_{j k}}\left|f_{i j}(\mathbf{x})-f_{i j}(\mathbf{x})\right| M_{\varepsilon, i}(\mathbf{x}) d S_{i j}(\mathbf{x}) .
\end{aligned}
$$

The proof is completed by Lemma 3.1 .

Based on the previous lemma, we obtain the following super-exponential estimate for the equilibrium process.

Proposition 3.4. For all $c_{1}, c_{2} \in\{1,2\}, \delta>0,0 \leq t_{1}<t_{2} \leq T$, and $h \in$ $C^{1}(\mathbb{T})$, we have that

$$
\begin{aligned}
& \limsup _{\varepsilon \rightarrow 0} \limsup _{N \rightarrow \infty} \frac{1}{N} \log \mathbb{P}_{N}^{\mathrm{eq}}\left[\mid \frac{1}{N} \sum_{i \in \mathcal{T}_{c_{1}}^{N}} \int_{t_{1}}^{t_{2}} h\left(z_{i}^{N}(t)\right)\left[d A_{i, c_{2}}^{N}(t)\right.\right. \\
& \left.\left.-\rho_{i, c_{2}}^{N, \varepsilon}\left(\mathbf{x}^{N}(t)\right) d t\right] \mid>\delta\right] \\
& \quad=-\infty .
\end{aligned}
$$

ProOF. Denote simply by $E(N, h, \varepsilon, \delta)$ the event inside the bracket of (3.22). By Chebyshev's inequality, for any $a>0$,

$$
\begin{aligned}
\frac{1}{N} \log \mathbb{P}_{N}^{\mathrm{eq}}[E(N, h, \varepsilon, \delta)] & \\
\leq & -a \delta+\frac{1}{N} \log \mathbb{E}_{N}^{\mathrm{eq}} \exp \left\{a \sum_{i \in \mathcal{T}_{c_{1}}^{N}} \mid \int_{t_{1}}^{t_{2}} h\left(z_{i}^{N}(t)\right)\left[d A_{i, c_{2}}^{N}(t)\right.\right. \\
& \left.\left.-\rho_{i, c_{2}}^{N, \varepsilon}\left(\mathbf{x}^{N}(t)\right) d t\right] \mid\right\} .
\end{aligned}
$$


Let

$$
\mathcal{E}_{N}=\left\{\mathbf{e}=\left(e_{1}, e_{2}, \ldots, e_{N}\right): e_{i}= \pm 1 \forall 1 \leq i \leq N\right\} .
$$

By the inequality $e^{|x|} \leq e^{x}+e^{-x}$ and Feynman-Kac's formula, we can bound the last expectation by

$$
\begin{aligned}
\sum_{\mathbf{e} \in \mathcal{E}_{N}} & \mathbb{E}_{N}^{\mathrm{eq}} \exp \left\{a \sum_{i \in \mathcal{T}_{c_{1}}^{N}} e_{i} \int_{t_{1}}^{t_{2}} h\left(z_{i}^{N}(t)\right)\left[d A_{i, c_{2}}^{N}(t)-\rho_{i, c_{2}}^{N, \varepsilon}\left(\mathbf{x}^{N}(t)\right) d t\right]\right\} \\
& \leq \sum_{\mathbf{e} \in \mathcal{E}_{N}} \exp \left\{\left(t_{2}-t_{1}\right) \lambda_{h, N, \varepsilon, a, \mathbf{e}}\right\},
\end{aligned}
$$

where $\lambda_{h, N, \varepsilon, a, \mathbf{e}}, \mathbf{e}=\left(e_{1}, \ldots, e_{N}\right) \in \mathcal{E}_{N}$, is the largest eigenvalue of the operator

$$
\mathscr{L}_{N}+\frac{a}{N} \sum_{i \in \mathcal{T}_{c_{1}}^{N}, j \in \mathcal{T}_{c_{2}}^{N}} e_{i} h\left(z_{i}\right)\left\{\iota_{\varepsilon}\left(x_{j}-x_{i}\right)-\delta\left(x_{j}-x_{i}\right)\right\} .
$$

Assume now that, for all $\mathbf{e} \in \mathcal{E}_{N}$,

$$
\lambda_{h, N, \varepsilon, a, \mathbf{e}} \leq C N\left[a\left(\varepsilon^{\frac{1}{4}}+N^{-\frac{1}{2}}\right)+a^{8}\left(\varepsilon^{\frac{1}{4}}+N^{-\frac{1}{2}}\right)^{8}\right]
$$

for some constant $C$ which depends only on $h$. Then we can deduce from (3.23), (3.24) and (3.25) that

$$
\begin{aligned}
& \frac{1}{N} \log \mathbb{P}_{N}^{\mathrm{eq}}[E(N, h, \varepsilon, \delta)] \\
& \quad \leq-a \delta+\log 2+\left[a\left(\varepsilon^{\frac{1}{4}}+N^{-\frac{1}{2}}\right)+a^{8}\left(\varepsilon^{\frac{1}{4}}+N^{-\frac{1}{2}}\right)^{8}\right]
\end{aligned}
$$

and, therefore, the left-hand side of (3.22) is bounded above by $-a \delta+\log 2$. This completes the proof since $a$ is an arbitrary positive number. Thus, the proof of proposition is reduced to the verification of (3.25). To this end, recall (cf. [18], Section 7) that the variational formula for $\lambda_{h, N, \varepsilon, a, \mathbf{e}}$ is $\sup _{f \in \mathscr{P}_{N}}$ of

$$
\begin{aligned}
& -\mathcal{D}_{N}(f)+\frac{a}{N} \sum_{i \in \mathcal{T}_{c_{1}}^{N}, j \in \mathcal{T}_{c_{2}}^{N}} e_{i}\left[\int_{G_{N}} h\left(z_{i}\right) f(\mathbf{x}) \iota_{\varepsilon}\left(x_{j}-x_{i}\right) d x\right. \\
& \left.-\frac{1}{2} \int_{F_{i j}} h\left(z_{i}\right)\left(f_{i j}(\mathbf{x})+f_{j i}(\mathbf{x})\right) d S_{i j}(\mathbf{x})\right] .
\end{aligned}
$$

By Lemma 3.2, we can bound this expression above by

$$
N\left[-\left(\mathcal{D}_{N}(f) / N\right)+C a\left(1+\left[\mathcal{D}_{N}(f) / N\right]^{\frac{7}{8}}\right)\left(\varepsilon^{\frac{1}{4}}+N^{-\frac{1}{2}}\right)\right] .
$$

Hence, we can prove (3.25) by an elementary inequality $a^{7 / 8} b-a \leq(7 b / 8)^{8}$ for $a, b>0$.

By the argument presented in (3.7), we obtain the following corollary. Notice that the test function $h$ depends only on the spatial variable. 
Corollary 3.3. For all $c_{1}, c_{2} \in\{1,2\}, \delta>0,0 \leq t_{1}<t_{2} \leq T$, and $h \in$ $C^{1}(\mathbb{T})$

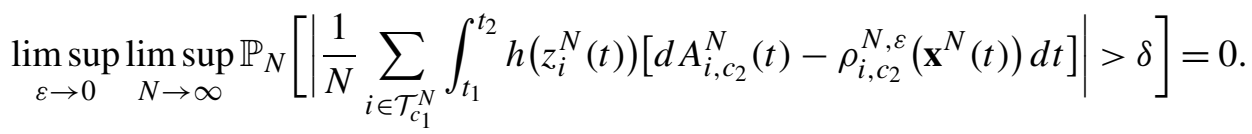

We now prove Theorem 3.1 .

Proof of TheOREM 3.1. For any $\eta>0$, we can find a partition $0=t_{0}<$ $t_{1}<\cdots<t_{M+1}=T$ of $[0, T]$ so that the function

$$
\widetilde{h}(t, x)=\sum_{i=0}^{M} \mathbb{1}_{\left[t_{i}, t_{i+1}\right]}(t) h\left(t_{i}, x\right)
$$

satisfies $\|\widetilde{h}-h\|_{\infty}<\eta$. By Corollary 3.3, the statement of theorem holds for $\widetilde{h}$. Since $\eta>0$ is arbitrary, it suffices to verify that

$$
\underset{M \rightarrow \infty}{\limsup \limsup \sup _{N \rightarrow \infty}} \mathbb{P}_{N}\left[\left|\frac{1}{N} \sum_{i \in \mathcal{T}_{c_{1}}^{N}} A_{i, c_{2}}^{N}(T)\right|>M\right]=0,
$$

(3.27) $\limsup _{M \rightarrow \infty} \limsup _{\varepsilon \rightarrow 0} \limsup _{N \rightarrow \infty} \mathbb{P}_{N}\left[\left|\frac{1}{N} \sum_{i \in \mathcal{T}_{c_{1}}^{N}} \int_{0}^{T} \rho_{i, c_{2}}^{N, \varepsilon}\left(\mathbf{x}^{N}(t)\right) d t\right|>M\right]=0$.

For (3.26), it is enough to prove that

$$
\mathbb{E}_{N}\left[\frac{1}{N^{2}} \sum_{i \neq j} A_{i j}^{N}(t)\right] \leq C
$$

for some constant $C$ depending only on $T$. Note that the last expectation can be written as

$$
\frac{t}{N^{2}} \sum_{i \neq j} \int_{F_{i j}}\left(\bar{f}_{N}^{[0, t]}\right)_{i j}(\mathbf{x}) d S_{i j}(\mathbf{x}) .
$$

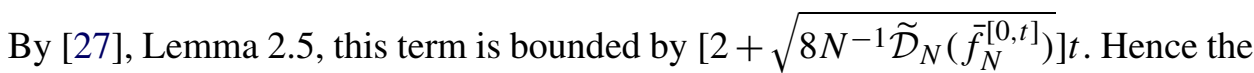
proof of (3.28) can be completed by (3.5) and Corollary 3.1.

For (3.27), observe first that

$$
\begin{aligned}
\mathbb{P}_{N}\left[\left|\frac{1}{N} \sum_{i \in \mathcal{T}_{c_{1}}^{N}} \int_{0}^{T} \rho_{i, c_{2}}^{N, \varepsilon}\left(\mathbf{x}^{N}(t)\right) d t\right|>M\right] \\
\leq \mathbb{P}_{N}\left[\left|\frac{1}{N} \sum_{i \in \mathcal{T}_{c_{1}}^{N}} A_{i, c_{2}}^{N}(T)\right|>\frac{M}{2}\right] \\
\quad+\mathbb{P}_{N}\left[\left|\frac{1}{N} \sum_{i \in \mathcal{T}_{c_{1}}^{N}}\left(A_{i, c_{2}}^{N}(T)-\int_{0}^{T} \rho_{i, c_{2}}^{N, \varepsilon}\left(\mathbf{x}^{N}(t)\right) d t\right)\right|>\frac{M}{2}\right] .
\end{aligned}
$$


Then two probabilities can be controlled respectively by (3.26), and by Corollary 3.3 with $h \equiv 1, t_{1}=0$ and $t_{2}=T$, respectively.

3.7. Proof of Theorem 2.1. In the one-component system, the limit of uncolored empirical density is obtained by the solution of the heat equation. By similar computation, we can derive the following lemma.

LEMma 3.3. Let $\mathbb{Q}_{\infty}$ be a weak limit of $\left\{\mathbb{Q}_{N}\right\}_{N=1}^{\infty}$. Then $\mathbb{Q}_{\infty}$ is concentrated on the trajectory of the form $\left(\rho_{1}(\cdot, x) d x, \rho_{2}(\cdot, x) d x\right)^{\dagger}$, where $\left(\rho_{1}, \rho_{2}\right)$ weakly satisfies

$$
\frac{\partial}{\partial t}\left[\frac{\rho_{1}(t, x)}{\sigma_{1}^{2}}+\frac{\rho_{2}(t, x)}{\sigma_{2}^{2}}\right]=\frac{1}{2} \rho_{x x}(t, x) .
$$

Proof. For any $f \in C^{1,2}([0, T] \times \mathbb{T})$, by (2.8) and (2.9), we can check that

$$
\begin{gathered}
\frac{1}{N} \sum_{i=1}^{N}\left[\frac{1}{\sigma_{c(i)}^{2}} f\left(t, x_{i}^{N}(t)\right)-\frac{1}{\sigma_{c(i)}^{2}} f\left(0, x_{i}^{N}(0)\right)\right. \\
\left.-\int_{0}^{t}\left(f_{t}+\frac{1}{2} f_{x x}\right)\left(s, x_{k}^{N}(s)\right) d s\right]
\end{gathered}
$$

is a martingale, and can be expressed as

$$
\frac{1}{N} \sum_{i=1}^{N} \frac{1}{\sigma_{c(i)}} \int_{0}^{t} f_{x}\left(s, x_{i}^{N}(s)\right) d \beta_{i}(s):=M_{f}(t) .
$$

Since $\mathbb{E}_{N}\left[M_{f}^{2}(t)\right]=O\left(N^{-1}\right)$, the expression (3.31) converges to 0 in probability as $N \rightarrow \infty$. This completes the proof.

ProOF OF THEOREM 2.1. We start by applying Itô's formula to the density field of $\left\{z_{i}^{N}(\cdot)\right\}_{i \in \mathcal{T}_{1}^{N}}$. For $f \in C^{1,2}([0, T] \times \mathbb{T})$, we can write

$$
\frac{1}{N} \sum_{i \in \mathcal{T}_{1}^{N}}\left[f\left(t, z_{i}^{N}(t)\right)-f\left(0, z_{i}^{N}(0)\right)\right]=J_{1}(t)+J_{2}(t)+J_{3}(t)
$$

where

$$
\begin{aligned}
& J_{1}(t)=\frac{1}{N} \sum_{i \in \mathcal{T}_{1}^{N}} \int_{0}^{t} f_{t}\left(s, z_{i}^{N}(s)\right) d s \\
& J_{2}(t)=\frac{1}{2 N} \sum_{i \in \mathcal{T}_{1}^{N}} \int_{0}^{t} f_{x x}\left(s, z_{i}^{N}(s)\right) d\left\langle z_{i}^{N}, z_{i}^{N}\right\rangle_{s}, \\
& J_{3}(t)=\frac{1}{N} \sum_{i \in \mathcal{T}_{1}^{N}} \int_{0}^{t} f_{x}\left(s, z_{i}^{N}(s)\right) d z_{i}^{N}(s) .
\end{aligned}
$$


We first demonstrate that the martingale $J_{3}(t)$ is negligible. By (3.14), (3.16) and (3.28),

$$
\mathbb{E}_{N}\left[J_{3}^{2}(t)\right] \leq C N^{-1} t+C N^{-1} \mathbb{E}_{N}\left[N^{-2} \sum_{i \neq j} A_{i j}^{N}(t)\right]=O\left(N^{-1}\right) .
$$

Thus, $J_{3}(t)$ is negligible by Doob's inequality.

We now consider the quadratic variation part in $J_{2}(t)$. By (3.16) and Theorem 3.1, we are able to approximate $d\left\langle z_{i}^{N}, z_{i}^{N}\right\rangle_{s}$ by

$$
\lambda \alpha^{2} \sigma_{c(i)}^{2}\left[\lambda+\frac{\mu_{1}^{N}(s) * \iota_{\varepsilon}\left(x_{i}^{N}(s)\right)}{\sigma_{1}^{2}}+\frac{\mu_{2}^{N}(s) * \iota_{\varepsilon}\left(x_{i}^{N}(s)\right)}{\sigma_{2}^{2}}\right] d s .
$$

For $\rho_{1}, \rho_{2} \in L^{2}(\mathbb{T})$, define

$$
F(t, x)=F_{\rho_{1}, \rho_{2}}(t, x)=x+\alpha \int_{\mathbb{T}} v(y-x)\left(\rho_{1} / \sigma_{1}^{2}+\rho_{2} / \sigma_{2}^{2}\right)(t, y) d y .
$$

Note that we can rewrite $(3.12)$ as

$$
\begin{aligned}
z_{i}^{N}(t)= & x_{i}^{N}(t)+\left(\alpha / \sigma_{1}^{2}\right)\left\langle\mu_{1}^{N}(t), v\left(\cdot-x_{i}^{N}(t)\right)\right\rangle \\
& +\left(\alpha / \sigma_{2}^{2}\right)\left\langle\mu_{2}^{N}(t), v\left(\cdot-x_{i}^{N}(t)\right)\right\rangle .
\end{aligned}
$$

If we replace $\mu_{c}^{N}(t), c \in\{1,2\}$, with $\rho_{c}(t, x) d x$, then the right-hand side becomes $F\left(t, x_{i}^{N}(t)\right)$. By combining this observation, (3.32), (3.33), (3.34), (3.35) and Proposition 3.3, we can conclude that, for any $\delta>0$,

$$
\begin{aligned}
\limsup _{\varepsilon \rightarrow 0} & \mathbb{Q}_{\infty}\left[\left(\rho_{1}(\cdot, x) d x, \rho_{2}(\cdot, x) d x\right)^{\dagger}:\right. \\
& \mid \int_{\mathbb{T}} f(T, F(T, x)) \rho_{1}(T, x) d x-\int_{\mathbb{T}} f(0, F(0, x)) \rho_{1}(0, x) d x \\
& \left.-\int_{0}^{T} \int_{\mathbb{T}}\left[f_{t}+\frac{\lambda \alpha^{2} \sigma_{1}^{2}}{2}\left(\lambda+\frac{\rho_{1} * \iota_{\varepsilon}}{\sigma_{1}^{2}}+\frac{\rho_{2} * \iota_{\varepsilon}}{\sigma_{2}^{2}}\right) f_{x x}\right] \rho_{1} d x d s \mid>\delta\right]=0,
\end{aligned}
$$

where $f_{t}$ and $f_{x x}$ are evaluated at $(s, F(s, x))$, while $\rho_{c} * \iota_{\varepsilon}=\rho_{c}(s, \cdot) * \iota_{\varepsilon}$ is evaluated at $(s, x)$. By Proposition 3.3, we know that $\rho_{c} * \iota_{\varepsilon}$ converges to $\rho_{c}$ in $L^{2}$ as $\varepsilon \rightarrow 0$ and, therefore, we obtain from (3.36) that

$$
\begin{aligned}
\mathbb{Q}_{\infty}[ & \left(\rho_{1}(\cdot, x) d x, \rho_{2}(\cdot, x) d x\right)^{\dagger}: \\
& \int_{\mathbb{T}} f(T, F(T, x)) \rho_{1}(T, x) d x-\int_{\mathbb{T}}(0, F(0, x)) \rho_{1}(0, x) d x \\
& \left.-\int_{0}^{T} \int_{\mathbb{T}}\left[f_{t}+\frac{\lambda \alpha^{2} \sigma_{1}^{2}}{2}\left(\lambda+\frac{\rho_{1}}{\sigma_{1}^{2}}+\frac{\rho_{2}}{\sigma_{2}^{2}}\right) f_{x x}\right] \rho_{1} d x d s=0\right]=0 .
\end{aligned}
$$


As before, $f_{t}$ and $f_{x x}$ are evaluated at $(s, F(s, x))$, and $\rho_{1}$ and $\rho_{2}$ are evaluated at $(s, x)$. Now we wish to replace $f(t, F(t, x))$ with $g(t, x) \in C^{1,2}([0, T] \times \mathbb{T})$ to complete the derivation of equation (2.14). To this end, first observe that, for any $h \in L^{1}(\mathbb{T})$,

$$
\frac{d}{d x}\left[\int_{\mathbb{T}} v(y-x) h(y) d y\right]=h(x)-\int_{\mathbb{T}} h(y) d y .
$$

Hence, we can write $F_{x}(t, x)=\alpha U(t, x)$ where

$$
U(t, x)=\lambda+\rho_{1}(t, x) / \sigma_{1}^{2}+\rho_{2}(t, x) / \sigma_{2}^{2}>\lambda>0 .
$$

Thus, there exists the inverse function $G(t, \cdot)$ of $F(t, \cdot)$ so that $F(t, G(t, x))=$ $G(t, F(t, x))=x$ for all $t$ and $x$. Now we can insert $f(t, x)=g(t, G(t, x))$, which implies $f(t, F(t, x))=g(t, x)$.

At this moment, we need that $g \in C^{1,2}$, while our computations leading to (3.37) requires $f \in C^{1,2}$ and, therefore, we should have $f, g \in C^{1,2}$ simultaneously. This is guaranteed if $\rho_{1}, \rho_{2} \in C^{1,2}$. Hence, we first assume that $\rho_{1}, \rho_{2}$ are smooth $x$. For this case, we can check $F_{x x}=\alpha U_{x}$ and $F_{t}=\frac{\alpha}{2} \rho_{x}$ where the latter follows from Lemma 3.3. Hence, by elementary computations we can check

$$
G_{x}(t, x)=\frac{1}{\alpha U(t, F(t, x))}, \quad G_{x x}(t, x)=-\frac{U_{x}(t, F(t, x))}{\alpha^{2} U^{3}(t, F(t, x))},
$$

and

$$
G_{t}(t, x)=-\frac{\rho_{x}(t, F(t, x))}{2 U(t, F(t, x))} .
$$

Finally, substitute $f(t, x)$ in (3.37) by $g(t, G(t, x))$ to obtain

$$
\begin{aligned}
& \mathbb{Q}_{\infty}\left[\left(\rho_{1}(\cdot, x) d x, \rho_{2}(\cdot, x) d x\right)^{\dagger}:\right. \\
& \quad \int_{\mathbb{T}} g(T, x) \rho_{1}(T, x) d x-\int_{\mathbb{T}} f(0, x) \rho_{1}(0, x) d x \\
& \left.\quad-\int_{0}^{T} \int_{\mathbb{T}}\left[g_{t}-\frac{\rho_{x} U+\lambda \sigma_{1}^{2} U_{x}}{2 U^{2}} g_{x}+\frac{\lambda \sigma_{1}^{2}}{2 U} g_{x x}\right] \rho_{1} d x d s=0\right]=1 .
\end{aligned}
$$

This completes the proof when $\rho_{1}, \rho_{2}$ are smooth. For the general case, we mollify $\rho_{1}, \rho_{2}$ by $\rho_{1} * \phi_{\eta}, \rho_{2} * \phi_{\eta}$ with the smooth mollifier $\left\{\phi_{\eta}\right\}_{\eta>0}$ and then apply the argument above to $\rho_{1} * \phi_{\eta}, \rho_{2} * \phi_{\eta}$. Ultimately, we allow $\eta \rightarrow 0$ to obtain (3.38). The precise argument of this procedure can be found in [27], Lemma 4.12.

In particular, (3.38) proves the first coordinate of (2.14), that is, the equation for $\rho_{1}$. The equation for $\rho_{2}$ can be proven in an identical manner. Therefore, the identification of limit points as the weak solution of (2.14) with initial condition $\widetilde{\rho}^{0}(x)$ is completed. tion.

We postpone the discussion of the uniqueness of weak solution to the next sec- 


\section{Uniqueness.}

4.1. Revisit: Two-color system. We start by considering the two-color system. At the time when this paper was written, there existed three limit theorems for the two-color system: SSEP [24], ZRP [7] and LIBM [27]. These three models share the same form of hydrodynamic limit. The evolution of the limiting particle densities $\rho_{1}, \rho_{2}$ of the two colors are obtained as the solution of the following PDE:

$$
\frac{\partial}{\partial t}\left(\begin{array}{c}
\rho_{1} \\
\rho_{2}
\end{array}\right)=\frac{1}{2} \nabla \cdot\left[\left(\begin{array}{ll}
\frac{\rho_{1}}{\rho} D(\rho)+\frac{\rho_{2}}{\rho} S(\rho) & \frac{\rho_{1}}{\rho}(D(\rho)-S(\rho)) \\
\frac{\rho_{2}}{\rho}(D(\rho)-S(\rho)) & \frac{\rho_{2}}{\rho} D(\rho)+\frac{\rho_{1}}{\rho} S(\rho)
\end{array}\right) \nabla\left(\begin{array}{c}
\rho_{1} \\
\rho_{2}
\end{array}\right)\right] .
$$

We briefly explain this equation:

- $\rho=\rho_{1}+\rho_{2}$ is the uncolored limiting density.

- $D(\rho)$ is the bulk-diffusion coefficient, that is, $\rho$ is the solution of

$$
\frac{\partial \rho}{\partial t}=\frac{1}{2} \nabla \cdot[D(\rho) \nabla \rho] .
$$

We can also derive this equation from (4.1) by simply adding two equations in (4.1). For instance, for the SSEP and the LIBM, $D(\rho) \equiv 1$, so that equation (4.2) becomes the heat equation. That is because the nature of interaction is reflection. For the ZRP, $D(\rho)$ is not a constant function and we refer to [18], Section 5, for details.

- $S(\rho)$ is the self-diffusion coefficient in the equilibrium with density $\rho$. The closed form of the self-diffusion coefficient is known only for LIBM [9] and ZRP [7]. In particular, for the LIBM, $S(\rho)=\frac{\lambda}{\lambda+\rho}$ where $\lambda$ is the interaction parameter. The closed form is not known for the SSEP but the regularity of $S(\cdot)$ has been established in [22].

This kind of universality is an interesting feature of the theory of interacting particle systems. In particular, we can derive the nonequilibrium behavior of the tagged particles, the so-called propagation of chaos [25], from the limit theorem for twocolor system. We emphasize here that the uniqueness of equation (4.1) is not a significant issue. This becomes obvious when we substitute $\rho_{2}=\rho-\rho_{1}$ in the first equation of (4.1) to obtain

$$
\frac{\partial \rho_{1}}{\partial t}=\frac{1}{2} \nabla \cdot\left[S(\rho) \nabla \rho_{1}+\frac{(D(\rho)-S(\rho)) \nabla \rho}{\rho} \rho_{1}\right] .
$$

Given that $\rho$ is the solution of the master equation (4.2), we can simply regard (4.3) as a linear parabolic PDE. Thus, under appropriate initial conditions and the nondegeneracy of $S(\cdot)$, the uniqueness is automatically guaranteed.

REMARK 4.1. We can observe an interesting property of the particle system from (4.2) and (4.3); the bulk evolution of particles of specific color is not governed by the bulk-diffusion coefficient $D(\cdot)$ but by the self-diffusion coefficient $S(\cdot)$ only. 
REMARK 4.2. An $m$-color system with $m \geq 3$ produce exactly the same result; (4.3) does not depend on the number of colors we used.

Now we consider equation (2.14) for the two-component system. In this case, due to the inhomogeneity of diffusivity of particles, the master equation (3.30), which corresponds to (4.2) for homogeneous system, cannot be solved by itself. Accordingly, an iterative strategy for solving the two-color system is unavailable and instead, we have to confront the system of equation (2.14) in a direct manner. Remark that the quasi-linear parabolic equation of the form (2.14) is known as a cross-diffusion equation.

4.2. Uniqueness. The general theory for a cross-diffusion equation was thoroughly explained in [1]. In this section, we use this general theory to develop the local uniqueness of (2.14).

Let us consider an $n$-dimensional quasi-linear equation

$$
\partial_{t} \mathbf{u}=\operatorname{div} \cdot[A(\mathbf{u}) \nabla \mathbf{u}],
$$

where $\mathbf{u}(\cdot)$ is an $n$-dimensional vector function and $A(\cdot)$ is an $n \times n$ matrix functional. In most physical situations of multi-component diffusive flow (e.g., [1, 3, $9,16,28]$ and models therein) the diffusion matrix $A(\mathbf{u})$ is neither symmetric nor positive semi-definite. Instead, it becomes evident that the physically relevant condition for $A(\mathbf{u})$ is the normal ellipticity. More precisely, a square matrix $M$ is called normally elliptic if all of its eigenvalues have a positive real part. Equation (4.4) is considered normally elliptic if $A(\mathbf{u})$ is normally elliptic for all $\mathbf{u}$. The normally elliptic parabolic equations differ intrinsically from the uniformly elliptic one. In particular, in [30], examples of normally elliptic equations without the maximum principle or even worse than that, equations that blow up in finite time, were suggested. In experimental physics (cf. [4]), this behavior of multi-component system has also been verified.

The normally elliptic equation has been analyzed in [1], in which the local existence and the uniqueness of solution were obtained. In particular, the following theorem is a direct consequence of [1], Theorems 14.4, 14.6 and 15.1.

THEOREM 4.1. Suppose that a function $\widetilde{\rho}^{0}$ satisfies Assumption 2.1. Then there exists $t^{+}\left(\widetilde{\rho}^{0}\right)>0$ such that equation (2.14) has a unique weak nonnegative solution $\left(\rho_{1}, \rho_{2}\right)^{\dagger}$ provided that $T<t^{+}\left(\widetilde{\rho}^{0}\right)$.

PROOF. The normal ellipticity of $D\left(\rho_{1}, \rho_{2}\right)$ of (2.15) is obvious since both of its trace and determinant are positive. We note here that the condition $\widetilde{\rho}^{0} \in$ $W_{1, p}(\mathbb{T})^{2}$ for some $p>2$ of Assumption 2.1 is used here for satisfying the requirement of [1], Theorem 14.4.

This theorem proves part (1) of Theorem 2.1. As we can anticipate from the peculiar behavior of the solution of certain examples in [30], the analysis of equation 
(4.4) is more delicate than the analysis of usual parabolic equations. In particular, the general theory established in [1], Theorem 14.4, requires a priori bound of $\sup _{t \geq 0}\|\widetilde{\rho}(t, \cdot)\|_{W_{k, p}}$ for some $k$ and $p$ to achieve the global uniqueness result. This bound for our model would be very difficult to obtain. We also stress here that the reference [30] demonstrate a counterexample for which the global uniqueness does not hold.

4.3. The Maxwell-Stefan equation. Equation (2.14) is not only an normally elliptic equation but also has some underlying physical structures, which may hopefully be exploited to develop a more concrete result than what we obtained in the previous section. In fact, in addition to the normal ellipticity, we observed that (2.14) is equivalent to the well-known Maxwell-Stefan equation [23]. We introduce the Maxwell-Stefan equation and refer to [2, 15, 16] for detailed exposition of this equation. In particular, [2] contains the physical derivation of the equation. Our outline follows mostly that of [16].

In principle, the Maxwell-Stefan equation describes the diffusive behavior of multi-component gaseous mixtures. Consider a system consisting of $n$ components and $u_{i}, 1 \leq i \leq n$, denotes the molar concentration of the $i$ th component. We assume $\sum_{i=1}^{n} u_{i} \equiv 1$ so that the dimension of equation is $n-1$. Denote by $J_{i}$ the flux of the $i$ th component, and assume $\sum_{i=1}^{n} J_{i} \equiv 0$. Finally, $D_{i j}=D_{j i}>0, i \neq j$, represents the constant binary diffusion coefficient between $i$ th and $j$ th components. Then the Maxwell-Stefan equation is given by

$$
\left\{\begin{array}{l}
\frac{\partial u_{i}}{\partial t}=-\nabla \cdot J_{i}, \\
\nabla u_{i}=-\sum_{j: j \neq i} \frac{u_{j} J_{i}-u_{i} J_{j}}{D_{i j}},
\end{array} \quad i=1,2, \ldots, n .\right.
$$

Note that, at least in principle, we can recover $\left(J_{1}, \ldots, J_{n}\right)$ as a function of $\left(u_{1}, \ldots, u_{n}\right)$ from the second equation, and by inserting this result into the first equation, we can derive an equation for $\left(u_{1}, \ldots, u_{n}\right)$. This procedure can be explicitly carried out for the ternary system, that is, $n=3$. For this case, by the elementary computation that we explained previously, it can be shown that $\mathbf{u}=\left(u_{1}, u_{2}\right)$ (recall that $u_{3}=1-u_{1}-u_{2}$ ) satisfies (4.4) with the cross diffusion matrix

$$
A\left(u_{1}, u_{2}\right)=\frac{1}{f\left(u_{1}, u_{2}\right)}\left(\begin{array}{cc}
D_{23}+\left(D_{12}-D_{23}\right) u_{1} & \left(D_{12}-D_{13}\right) u_{1} \\
\left(D_{12}-D_{23}\right) u_{2} & D_{13}+\left(D_{12}-D_{13}\right) u_{2}
\end{array}\right)
$$

where

$$
f\left(u_{1}, u_{2}\right)=D_{13} D_{23}+D_{13}\left(D_{12}-D_{23}\right) u_{1}+D_{23}\left(D_{12}-D_{13}\right) u_{2} .
$$

This kind of simple derivation procedure is invalid for $n \geq 4$; see [15], Section 2, for details.

The equation (2.14) is equivalent to the ternary Maxwell-Stefan equation described previously under the condition that $D_{12}>D_{13}, D_{23}$. To this end, first, for 
the given ternary Maxwell-Stefan equation, let $k>0$ be an arbitrary real number and let

$$
\begin{aligned}
& \rho_{1}=k D_{13}\left(D_{12}-D_{23}\right) u_{1}, \quad \rho_{2}=k D_{23}\left(D_{12}-D_{13}\right) u_{2}, \\
& \sigma_{1}^{2}=D_{13}^{-1}, \quad \sigma_{2}^{2}=D_{23}^{-1} \quad \text { and } \quad \lambda=k D_{13} D_{23} .
\end{aligned}
$$

Then $\rho_{1}$ and $\rho_{2}$ can be easily verified to satisfy equation (2.14). On the other hand, for the given hydrodynamic limit equation (2.14), let $D_{12}$ be any number larger than $\max \left\{\sigma_{1}^{-2}, \sigma_{2}^{-2}\right\}$, and let

$$
\begin{aligned}
u_{1} & =\left[\lambda\left(\sigma_{2}^{2} D_{12}-1\right)\right]^{-1} \rho_{1}, \quad u_{2}=\left[\lambda\left(\sigma_{1}^{2} D_{12}-1\right)\right]^{-1} \rho_{2}, \\
D_{13} & =\sigma_{1}^{-2}, \quad D_{23}=\sigma_{2}^{-2} .
\end{aligned}
$$

Then $u_{1}$ and $u_{2}$ can be observed to satisfy the ternary Maxwell-Stefan equation. Owing to the multi-component nature of our model, this equivalence is quite natural. Hence, we can reduce the uniqueness problem of equation (2.14) to that of the Maxwell-Stefan equation.

An important feature of the Maxwell-Stefan equation is its entropy structure as a consequence of the Onsager reciprocity. More precisely, the diffusion matrix (4.6) can be written as $A(\cdot, \cdot)=K(\cdot, \cdot) \chi(\cdot, \cdot)$ where $\chi$ is the Hessian of entropy functional, and $K$ is a positive-definite and symmetric matrix. The normal ellipticity of the diffusion matrix naturally follows from this structure. Recently, the cross-diffusion equations under the presence of the entropy structure have been investigated by several articles. For instance, the global existence of the weak solution and its exponential decay to the steady state is proven in [16], and the boundedness of this global solution is established in [15]. However, the global uniqueness of with a general initial condition is known to be a delicate problem (cf. [15], Section 6), and the global uniqueness of the Maxwell-Stefan equation for a general class of initial conditions is an open problem. At the time when this paper is written, the global uniqueness of the Maxwell-Stefan equation is known only for the near-equilibrium case by [17]. In our context, this result can be stated in the following manner.

THEOREM 4.2. Under Assumption 2.2, there exists a unique global weak solution of (2.14).

The proof along with the nontrivial way to derive the constant $\varepsilon\left(\lambda, \sigma_{1}, \sigma_{2}\right)$ is summarized in [2], Section 9.4. This proves the part (2) of Theorem 2.1.

Acknowledgments. The author would like to thank Professor S. R. S. Varadhan for numerous valuable discussions, and Professor Ansgar Jüngel of Vienna University of Technology for many helpful comments regarding the crossdiffusion equation, especially for the material in Section 4. The author also acknowledges the support from the Samsung scholarship. 


\section{REFERENCES}

[1] Amann, H. (1993). Nonhomogeneous linear and quasilinear elliptic and parabolic boundary value problems. In Function Spaces, Differential Operators and Nonlinear Analysis (Friedrichroda, 1992). Teubner-Texte Math. 133 9-126. Teubner, Stuttgart. MR1242579

[2] Bothe, D. (2011). On the Maxwell-Stefan approach to multicomponent diffusion. In Parabolic Problems. Progr. Nonlinear Differential Equations Appl. 80 81-93. Birkhäuser, Basel. MR3052573

[3] Boudin, L., GötZ, D. and GreC, B. (2010). Diffusion models of multicomponent mixtures in the lung. ESAIM Proc. 30 91-104.

[4] Duncan, J. and Toor, H. (1962). An experimental study of three component gas diffusion. AIChE Journal 8 38-41.

[5] Erignoux, C. (2016). Hydrodynamic limit for an active exclusion process. Available at arXiv:1608.04937.

[6] Feng, S., Grigorescu, I. and QuAStel, J. (2004). Diffusive scaling limit of mutually interacting particle systems. SIAM J. Math. Anal. 35 1512-1533.

[7] Gabrielli, D., Jona-Lasinio, G. and LANDim, C. (1996). Onsager reciprocity relations without microscopic reversibility. Phys. Rev. Lett. 77 1202-1205.

[8] Gabrielli, D., Jona-Lasinio, G. and LANDiM, C. (1999). Onsager symmetry from microscopic TP invariance. J. Stat. Phys. 96 639-652.

[9] Giovangigli, V. (1999). Multicomponent Flow Modeling. Birkhäuser, Boston, MA. MR1713516

[10] Grigorescu, I. (1999). Self-diffusion for Brownian motions with local interaction. Ann. Probab. 27 1208-1267. MR1733146

[11] GRIGORESCU, I. (1999). Uniqueness of the tagged particle process in a system with local interactions. Ann. Probab. 27 1268-1282.

[12] Guo, M. Z., Papanicolaou, G. C. and Varadhan, S. R. S. (1988). Nonlinear diffusion limit for a system with nearest neighbor interactions. Comm. Math. Phys. 118 31-59. MR0954674

[13] IKeda, N. and Watanabe, S. (1981). Stochastic Differential Equations and Diffusion Processes. North-Holland, Amsterdam.

[14] Itô, K. and McKean, H. P. (1965). Diffusion Processes and Their Sample Paths. Springer, New York.

[15] JÜNGEL, A. (2015). The boundedness-by-entropy principle for cross-diffusion systems. Nonlinearity 28 1963-2001.

[16] JÜngel, A. and Stelzer, I. V. (2013). Existence analysis of Maxwell-Stefan systems for multicomponent mixtures. SIAM J. Math. Anal. 45 2421-2440. MR3090648

[17] KaWASHIma, S. (1984). Systems of a hyperbolic-parabolic composite type, with applications to the equations of magnetohydrodynamics. Doctoral Thesis, Kyoto Univ.

[18] Kipnis, C. and Landim, C. (1999). Scaling Limits of Interacting Particle Systems. Grundlehren der Mathematischen Wissenschaften 320. Springer, Berlin. MR1707314

[19] Kipnis, C. and Varadhan, S. R. S. (1986). Central limit theorem for additive functionals of reversible Markov processes and applications to simple exclusions. Comm. Math. Phys. 104 1-19. MR0834478

[20] Komorowski, T., Landim, C. and Olla, S. (2012). Fluctuations in Markov Processes: Time Symmetry and Martingale Approximation. Grundlehren der Mathematischen Wissenschaften 345. Springer Science \& Business Media, Berlin.

[21] Komorowski, T., Olla, S. and Simon, M. (2016). Macroscpoic evolution of mechanical and thermal energy in a harmonic chain with random flip of velocities. Available at arXiv:1609.02413. 
[22] Landim, C., Olla, S. and Varadhan, S. R. S. (2001). Symmetric simple exclusion process: Regularity of the self-diffusion coefficient. Comm. Math. Phys. 224 307-321.

[23] Maxwell, C. (1866). On the dynamical theory of gases. Phil. Trans. R. Soc. London 157 $49-88$.

[24] Quastel, J. (1992). Diffusion of color in the simple exclusion process. Comm. Pure Appl. Math. 45 623-679. MR1162368

[25] Rezakhanlou, F. (1994). Propagation of chaos for symmetric simple exclusion. Comm. Pure Appl. Math. 117 943-957.

[26] Sasada, M. (2010). Hydrodynamic limit for two-component exclusion processes. Stochastic Process. Appl. 120 494-521.

[27] SeO, I. (2017). Large deviation principle for interacting Brownian motions. Comm. Pure Appl. Math. 70 203-288.

[28] Shigesada, N., Kawasaki, K. and Teramoto, E. (1979). Spacial segregation of interacting species. J. Theoret. Biol. 79 83-99.

[29] Simon, M. (2013). Hydrodynamic limit for the velociti-flip model. Stochastic Process. Appl. $1233623-3662$.

[30] Stara, J. and John, O. (1995). Some (new) counterexamples of parabolic systems. Comment. Math. Univ. Carolin. 36 503-510.

[31] Varadhan, S. R. S. (1991). Scaling limits for interacting diffusions. Comm. Math. Phys. 135 313-353.

[32] Varadhan, S. R. S. (1993). Nonlinear diffusion limit for a system with nearest neighbor interactions. II. In Asymptotic Problems in Probability Theory: Stochastic Models and Diffusions on Fractals (Sanda/Kyoto, 1990). Pitman Res. Notes Math. Ser. 283 75-128. Longman Sci. Tech., Harlow. MR1354152

Department of Mathematical SCIENCES

SEOUL NATIONAL UNIVERSITY

GWANAK-Ro 1, GWANAK-GU

SEOUL 08826

KOREA

E-MAIL: insuk.seo@snu.ac.kr 